\title{
Novel transcription factor Satb2 interacts with matrix attachment region DNA elements in a tissue-specific manner and demonstrates cell-type-dependent expression in the developing mouse CNS
}

\author{
Olga Britanova, ${ }^{1,2}$ Sergey Akopov, ${ }^{2}$ Sergey Lukyanov, ${ }^{2}$ Peter Gruss $^{3}$ and Victor Tarabykin ${ }^{1}$ \\ ${ }^{1}$ Department of Molecular Biology of Neuronal Signals, Max-Planck Institute for Experimental Medicine, 37075 Goettingen, \\ Germany \\ ${ }^{2}$ Shemiakin and Ovchinnikov Institute of Bioorganic Chemistry RAS, Miklukho-Maklaya 16/10, 117871 Moscow, Russia \\ ${ }^{3}$ Department of Molecular Cell Biology, Max-Planck Institute for Biophysical Chemistry, 37077 Goettingen, Germany
}

Keywords: brain, chromatin, mouse, neocortex, spinal cord, transcription factor

\begin{abstract}
Satb1 is a first cell-type-specific transcription factor of a novel type that functions as a regulator of the transcription of large chromatin domains. We identified a close homologue of Satb1, Satb2, in a cDNA subtraction screening in a search for genes controlling neural differentiation. Satb2 showed $61 \%$ amino-acid homology to Satb1. Satb2 and Satb1 expression was detected in different cell subpopulations of developing mouse CNS in a mutually exclusive manner. In the electrophoretic mobility shift assay we demonstrate that nuclear extracts from the embryonic day 18.5 mouse developing neocortex, in contrast to basal ganglia, contain a protein complex interacting with matrix attachment region DNA elements (MARs) with high affinity. Endogenous Satb2 protein is a part of this complex. In the developing neocortex Satb2 was detected largely in the superficial layers. In the developing spinal cord Satb2 expression marks a subpopulation of Lbx1-positive neurons dorsally and a subgroup of Isl1-positive neurons ventrally. In the Lbx1 mutants Satb2 expression is greatly reduced. We suggest that Satb2 may regulate differentiation of subsets of neurons at the level of higher order chromatin structure via binding to MARs.
\end{abstract}

\section{Introduction}

The tissue-specific regulation of gene expression is critical to the development and function of all higher eukaryotic organisms. Tissuespecific transcription is controlled by the presence of certain sets of transcription factors that activate or suppress the expression of a subset of genes in a given cell type. In addition, chromatin modification is involved in the regulation of gene activity (Fry \& Peterson, 2001). Recent studies suggest the importance of high-order chromatin structure in the regulation of gene expression (Schubeler et al., 2000). Cell-type-specific gene regulation, at the level of chromatin organization, is an important aspect of cellular differentiation. However, this remains poorly studied.

Recently, the first transcription factor of a novel type that seems to be involved in the regulation of tissue-specific organization of chromatin, Satb1, was identified. Satb1 is a nuclear protein that can bind to a core unwinding element within the matrix attachment DNA region (MAR) (Dickinson et al., 1992). Unlike classic transcription factors, which bind individual target genes to regulate transcription, Satb1, which binds to multiple sites where chromatin is fastened to form loop domains, may dictate the organization and structure of chromatin domains, thereby orchestrating the transcription potential of multiple genes. Satb1 ablation led to gene disregulation affecting multiple genes in T-cells (Alvarez et al., 2000). Gene knock-out studies have also demonstrated that Satb1 acts as a global regulator of thymocyte

Correspondence: Dr V. Tarabykin, as above.

E.mail: vtaraby@gwdg.de

Received 11 June 2004, revised 3 November 2004, accepted 15 November 2004 differentiation that provides sites for tissue-specific organization of DNA sequences and regulates region-specific histone modifications (Yasui et al., 2002; Cai et al., 2003). The closest homologue of Satb1, Satb2, has recently been identified as a gene mutated in human patients with cleft palate (FitzPatrick et al., 2003). It has also been demonstrated that SATB2 can enhance gene expression and that the protein can be modified by sumoylation (Dobreva et al., 2003).

In this study we characterized the expression of both Satb1 and Satb2 in the developing mouse CNS. Satb2 expression is largely confined to subsets of postmitotic cells in the developing CNS with the highest expression in the developing cerebral cortex. Satbl is also expressed in several regions of developing CNS, but their expression patterns do not overlap during development. We have also demonstrated that developing neocortex, but not basal ganglia, contains a nuclear protein complex that can bind nuclear MARs. Satb2 protein is a part of the complex. Our findings suggest that Satb2 and Satb1 represent a novel class of transcription regulators that control neuronal differentiation of distinct neuronal subtypes by targeting chromatin and regulating expression of multiple loci in a manner similar to Satb1 action within the T-cell lineage.

\section{Materials and methods}

\section{MAR DNA labelling}

PCR labelling of MAR probes was performed as described by Nikolaev et al. (1996). Probes for binding with nuclear extract were prepared as follows: MAR-containing fragments were PCR-amplified 
in a $50-\mu \mathrm{L}$ reaction volume using the corresponding plasmid as template and a library primer 5'-ACTGAGCTCGAGTATCCATGA ACA.

The PCR labelling was conducted in a final volume of $50 \mu \mathrm{L}$ containing $\sim 10 \mathrm{ng}$ of the template fragment, $20 \mu \mathrm{M}$ library primer for MAR fragments, $200 \mu \mathrm{M}$ each dCTP, dGTP and dTTP, $20 \mu \mathrm{M}$ dATP, $50 \mu \mathrm{Ci}\left[\alpha-{ }^{32} \mathrm{P}\right] \mathrm{dATP}$ (Obninsk, Russia), $3 \mathrm{~mm} \mathrm{MgCl}_{2}$, $10 \mathrm{mM}$ Tris $/ \mathrm{HCl}(\mathrm{pH} \mathrm{8.3)}$ and $50 \mathrm{mM} \mathrm{KCl}$. The reaction was proceeded as follows: $94{ }^{\circ} \mathrm{C}$ for $0.8 \mathrm{~min}, 50{ }^{\circ} \mathrm{C}$ for $0.6 \mathrm{~min}, 72{ }^{\circ} \mathrm{C}$ for $1 \mathrm{~min}$, for 18 cycles. Reaction products were extracted with phenol-chloroform, precipitated with ethanol and dissolved in TE buffer (10 mM Tris/HCl; $1 \mathrm{~mm}$ EDTA).

\section{Animals}

Wild-type and $l b x l$ knock-out mouse embryos were used to isolate tissue samples. All animal manipulations were carried out in accordance with German law and were approved by the Bezirksregierung Braunschweig

\section{Nuclear matrix preparation and in vitro binding of MARs}

Animals were killed by cervical dislocation. Cortical hemispheres and basal ganglia of embryonic day (E)18.5 embryos were surgically removed and used for nuclear extract isolation. Nuclear extracts were prepared from embryonic tissue by a high-salt extraction method
(Cockerill \& Garrard 1986). Binding of radioactively labelled MARs to the nuclear extract in vitro was carried out as described by Izaurralde et al. (1988).

\section{Generation of antibodies against Satb2 protein}

In order to produce antibody against Satb2 we generated two different peptides based on predicted protein sequence:

1. C-SSPSSSRTPQAKTSTPTT (453-470 amino acids)

2. C-QQSQPTKESSPPREEA (585-600 amino acids).

These peptides were coupled to BSA (Sigma, St Louis, MO, USA) via MBS (Pierce, Rockford, IL, USA). Coupled peptides were injected into rabbits for immunization. The serum was purified using affinity resin columns (Pierce) pretreated with peptides 1 or 2 . Purified serum was tested at different dilutions on cryosections in immunohistochemistry experiments and on Western blots with protein isolated from the cortical tissue. Both antibodies ( $\mathrm{Ab} 1$ and $\mathrm{Ab} 2$ ) gave positive signals with cortical protein extract on Western blots (see Fig. 2). Western blot analysis of the protein extracts from bacterial cells expressing Satb2 cDNA also produced a band of the expected size (data not shown).

\section{RT-PCR amplification of Satb2 cDNA fragment}

The cDNA fragment of the predicted protein KIA1034 (Satb2) was amplified with following primers:

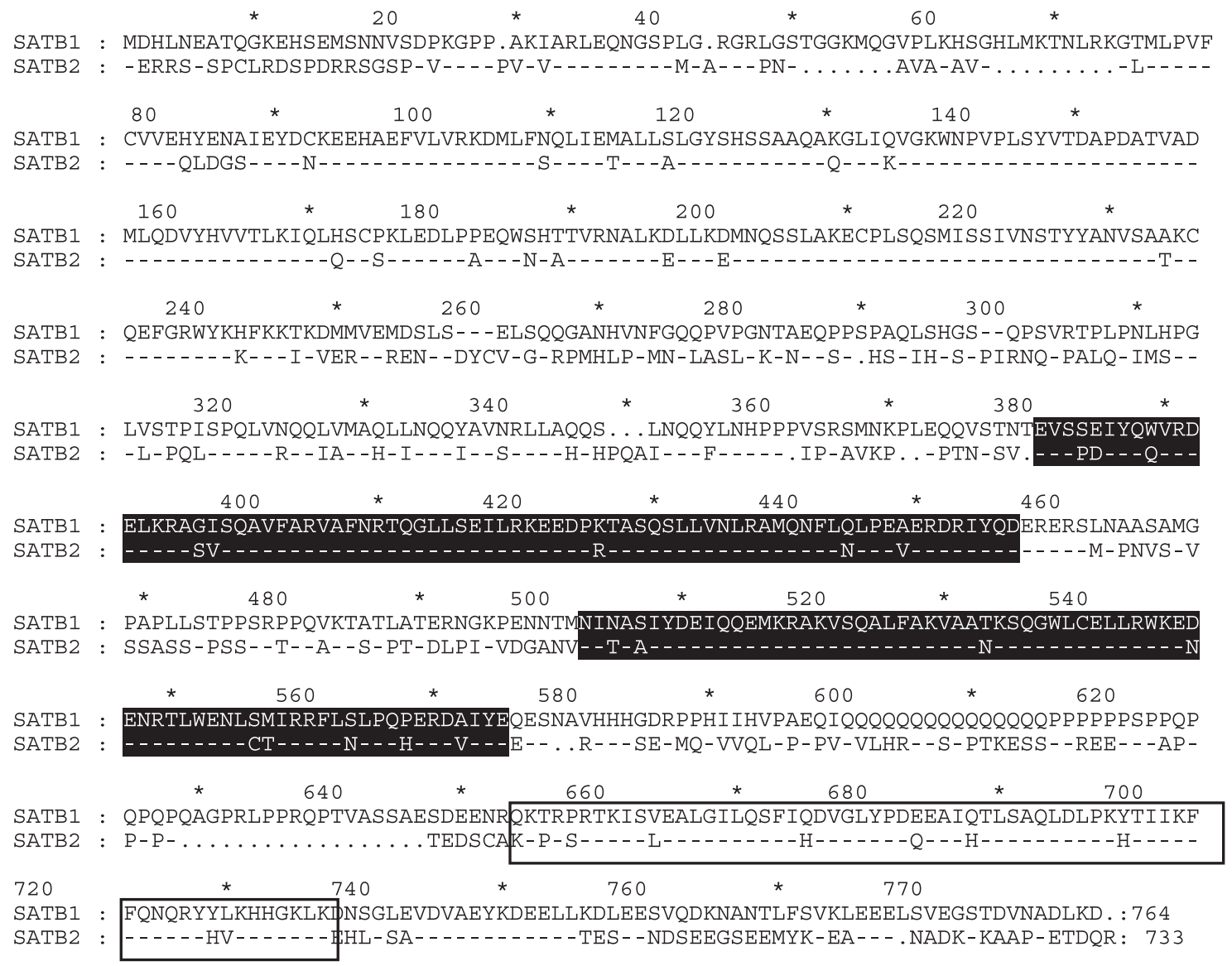

FIG. 1. Comparison of protein sequences for mouse Satb1 and Satb2. The numbers refer to the positions of amino acids. The black and open boxes denote the conserved 'Cut' and 'homeo'- domains. The dashes indicate identical amino acids. Dots indicate parts of one protein that do not have analogous amino acids in the other protein. 

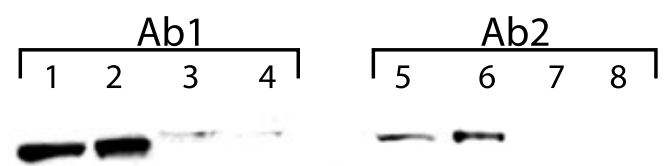

FIG. 2. Western blots with $\mathrm{Ab} 1$ and $\mathrm{Ab} 2$ anti-Satb2 antibodies. Lanes 1 and 5 contain total proteins and 2 and 6 nuclear extracts isolated from the E18.5 neocortex. Lanes 3 and 4 and 7 and 8 contain nuclear extracts isolated from the HeLa cells and E18.5 basal ganglia, respectively. Note that for each antibody there is a band of size close to the predicted size of $95 \mathrm{kDa}$ and an additional smaller band. The smaller bands, however, are not present in the nuclear extracts (lanes 2 and 6).

Satb2-dir 5'-TTATTGTCAGAGATACTGCGT (exon 7) Satb2-rev 5'-TTCTGTTGGAGGAGGCGGTG (exon 10)

The cortical cDNA from E15.5 mice was used as a template. The PCR product was cloned into the pGEM-T cloning system (Promega, Madison, WI, USA). For full cDNA amplification the following primers were designed:

Satb2-ATG 5'-ATGGAGCGGCGGAGCGAGAGC

Satb2-stop 5'-TTATCTCTGGTCGGTTTCGG.

\section{Immunohistochemistry}

Embryos were sectioned at $7-10 \mu \mathrm{m}$ with a cryostat (Leica), air dried for $20 \mathrm{~min}$, washed in PBS and fixed for $5 \mathrm{~min}$ in 4\% paraformaldehyde (PFA)/PBS. After three washes in PBS, sections were preblocked in $1 \%$ BSA $/ 0.1 \%$ Tween $20 / \mathrm{PBS}(1 \mathrm{~h})$, and incubated with primary antibodies overnight at $4{ }^{\circ} \mathrm{C}$ in the same solution. Sections were then washed in PBS and incubated with a diluted (1 : 1000) goat anti-rabbit secondary antibody (Molecular Probes) for $1 \mathrm{~h}$ at room temperature, rinsed with PBS and visualized under a fluorescence microscope. The working dilutions of the Satb2 antibodies were $1: 100$ for immunohistochemistry and $1: 1000$ for Western blotting.

The following antibodies were used: guinea-pig anti-Lbx1 (kindly provided by Dr C. Birchmayer), mAb Isl1/2 and Lim1/2 (4F2) (Developmental Studies Hybridoma Bank, University of Iowa, USA), guinea-pig anti-Lmx1b (kindly provided by Dr T. Jessell) and rabbit anti-Tbr1 (kindly provided Dr M. Sheng).

For BrdU birth-dating experiments, BrdU (100 mg/g body weight) (Sigma)/PBS was injected intraperitoneally at E11.5. Incorporated $\mathrm{BrdU}$ was detected with mouse anti-BrdU mAb (Sigma).

\section{In situ hybridization}

Radioactive in situ hybridization was performed as described by Stoykova \& Gruss (1994). As probes, coding and non-coding regions
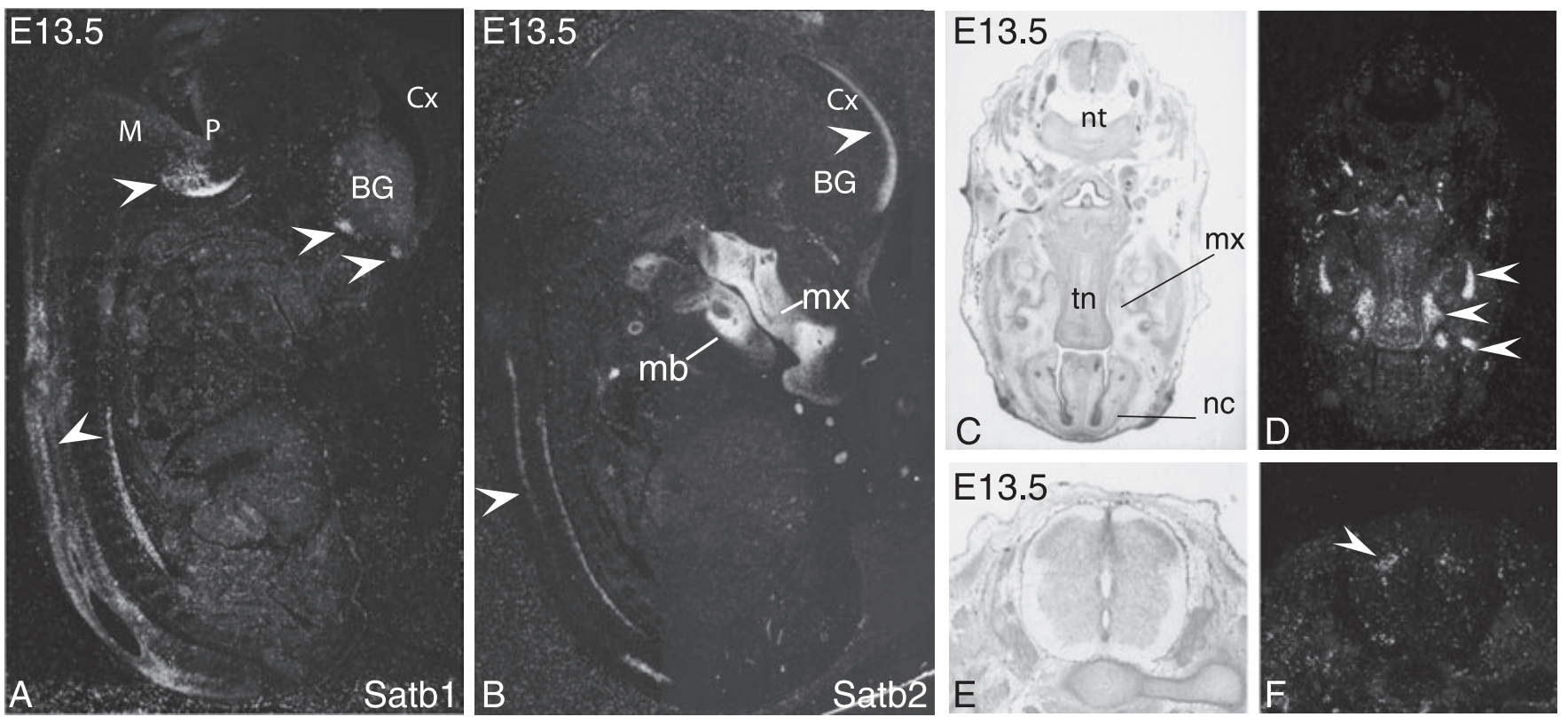

FIG. 3. Satb2 and Satb1 mRNA distribution in the developing mouse embryo at E13.5. (A, B, D and F) Dark-field images; (C and E) bright-field images. At E13.5 Satb2 and Satb1 are expressed in a mutually exclusive manner (A and B). Satb2 is mostly expressed in the neocortex and in the maxilla and mandible (B and D) and in the spinal cord (F; for spinal cord expression see Results). Satb1 is largely expressed in the spinal cord, in the transition field between medulla and pons and in two groups of cells in the ventral telencephalon (A). Main sites of expression are depicted by arrowheads. Abbreviations: BG, basal ganglia; m, medulla; mx, maxillar component; nc, nasal capsule; nt, notochord; $\mathrm{p}$, pons; tn, tongue

FIG. 4. Comparative expression of Satb2 at E15.5 in the forebrain. (A, G, J and L) Dark field images; (B-F, H, I, K, M and O) bright-field images. (H and I) Comparison of Satb2 expression (B) with that of Svet1 (C), Pax6 (D), Dlx1 (E) and Satb1 (F). G-I and J-L are high-magnification views of the boxed regions from A-C. The dashed lines in G, H and I demarcate the limits of the SVZ. The dashed lines in J and L demarcate the limits of Satb2 and Satb1 expression in the piriform cortex. (O) Satb2 mRNA distribution in the adult brain. Abbreviations: BG, basal ganglia; cp, cortical plate; iz, intermediate zone; SVZ, subventricular zone; pcx, piriform cortex; VZ, ventricular zone. 

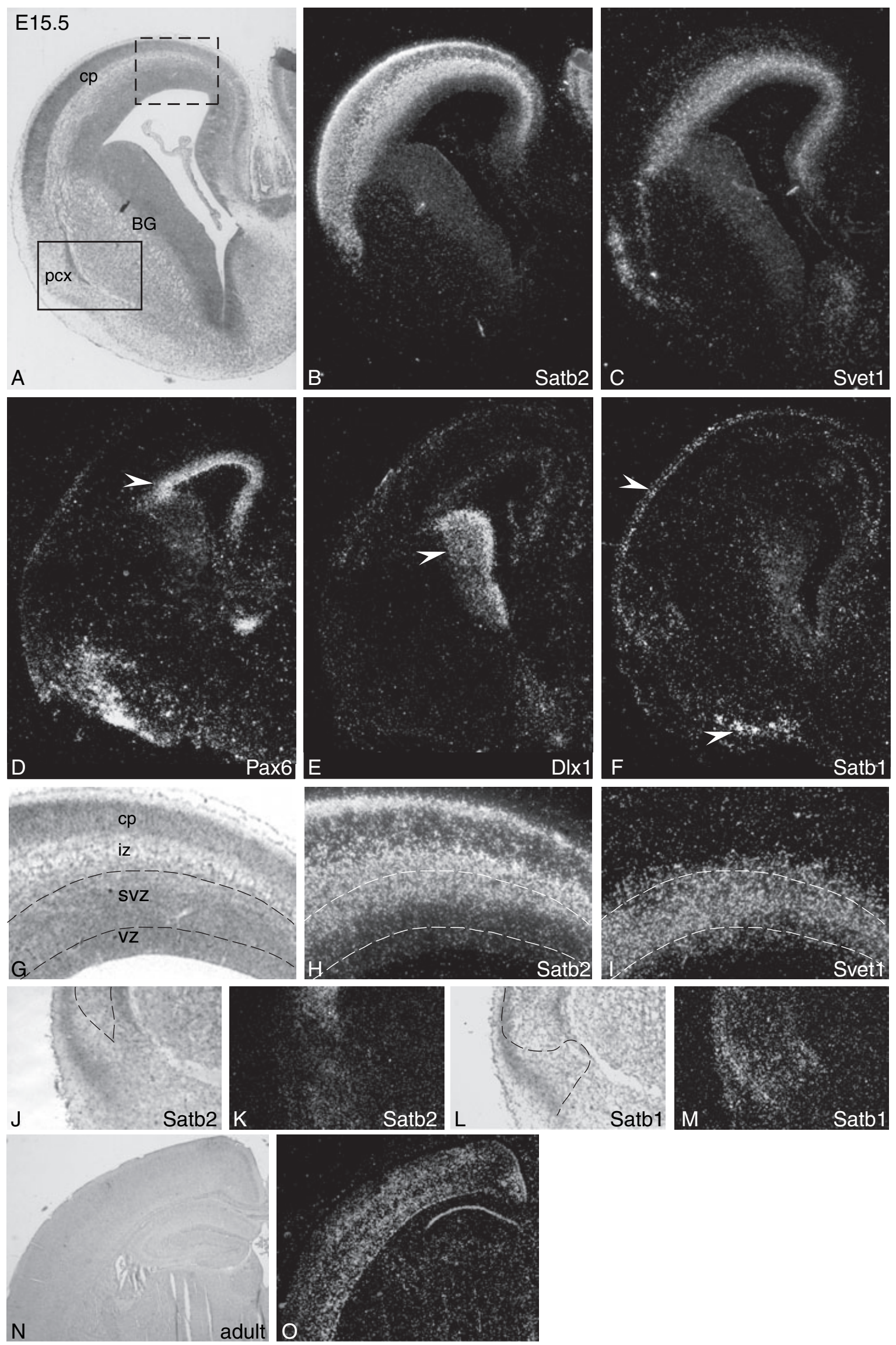

Satb1 M

Satb1 
were used. The coding region was amplified with primers Satb2-dir and Satb2-rev (see above).

\section{Results \\ Identification and structural characterization of a novel Satb1-related homeobox gene}

In order to identify genes involved in the control of mouse neocortical differentiation we performed a subtractive hybridization of mouse E15.5 PCR-amplified neocortical cDNA against that of E13.5 (Rebrikov et al., 2000). From this cDNA subtraction we selected several genes that were up-regulated in the neocortical subventricular zone (SVZ) at E15.5. A novel cDNA of $1.7 \mathrm{~kb}$ (clone A11) showed strong expression in the upper part of the cortical SVZ at E15.5 stage (see below).

In order to isolate a full cDNA copy of the corresponding gene we applied a modified 5'-RACE technology (Matz et al., 1999) that allowed us to isolate $9.6 \mathrm{~kb}$ of the A11 clone upstream region. This region did not contain any significant open reading frame, indicating that the cloned part of the gene belonged to either the $3^{\prime} / 5^{\prime}$ untranslated region (UTR) or was an intronic part of the underspliced RNA captured in the process of cDNA preparation. The latter hypothesis was confirmed after analysis of the Celera database. The isolated 9.6-kb sequence showed $100 \%$ homology to an intronic part of a gene KIA1034 identified in the Kazusa cDNA sequencing project (Kikuno et al., 2000). In order to confirm the expression pattern of KIA1034, we amplified a 538-bp part of a coding region from exons 7-10 of KIA1034 by PCR from the mouse E15.5 neocortical cDNA. This cloned fragment was used as a probe for in situ hybridization on paraffin sections. It produced an expression pattern identical to that of clone A11, indicating that A11 was a cDNA product of underspliced KIA1034 mRNA.

The KIA1034-predicted cDNA encoded a 733-amino-acid protein of $85.5 \mathrm{kDa}$. A GenBank protein homology search revealed that the Satb1 homeobox-containing gene was its closest homologue. We referred to the gene therefore as Satb2. Alignment of the Satb1 and Satb2 amino acid sequences showed an identity of $61 \%$ and similarity of $73 \%$ (Fig. 1). Both proteins have two 'cut' domains and an atypical homeodomain. A search of the GenBank database did not reveal anything other than Satb1 homologues, indicating that Satb1 and Satb2 are the only members of Satb subfamily of cut domain homeobox genes in the mammalian genome.

\section{Satb2 antibody production and specificity verification}

In order to extend the analysis of the Satb2 protein two antisera were raised in rabbits. The first, $A b 1$, was directed towards the peptide localized between amino acids 453 and 470 of the protein and the other, Ab2, was directed towards the peptide localized at positions
585-600 (Fig. 1, see also Materials and methods). Both peptide sequences were selected in the regions of the protein that did not show any homology to Satb1 and were unique for Satb2. In order to test these two antisera, Satb2 recombinant protein was produced in Escherichia coli. Western blots probed with Ab1 or Ab2 strongly revealed the protein (data not shown). Both antibodies were then tested for specificity by Western blotting on homogenates of embryonic neocortex, basal ganglia and HeLa cells (Fig. 2). Two protein bands were recognized by both antibodies. The heavier bands, with an apparent molecular weight of approximately $95 \mathrm{kDa}$, were identical for both antibodies (the predicted molecular weight of the Satb2 protein is $85.5 \mathrm{kDa}$ ). Two other bands had a size of $30 \mathrm{kDa}$ for $\mathrm{Ab} 1$ and $50 \mathrm{kDa}$ for $\mathrm{Ab} 1$. These bands did not appear in the cortical nuclear extracts, indicating that the corresponding cross-reacting antigens were present in the cytoplasm but not in the nucleus (Fig. 2).

We also performed immunohistochemical experiments on frozen and paraffin sections of embryos or brains taken from different stages of gestation. Both antibodies produced nuclear staining typical for a transcription factor (see below). The signal was always detected in the tissues where Satb2 RNA was detected too (see description of Satb2 protein expression below).

All these results indicate that both antibodies specifically recognize Satb2 in the nuclear extracts and nuclei in the tissue.

\section{Developmental distribution of Satb2 mRNA and protein suggests a role in neuronal differentiation}

The tissue distribution of Satb2 RNA and protein was analysed by radioactive in situ hybridization and immunohistochemistry (Figs 36). We also compared Satb2 expression with that of Satb1 and several other molecular markers. The first site of Satb2 expression was detected at E11.5 within the mandibular and maxillar components of the first developing brachial arch (Fig. 5A and B). At E13.5 Satb2 mRNA was additionally detected in the telencephalon, tongue muscles and in a subpopulation of dorsal spinal cord neurons (Fig. 3B, D and F). Satb1 expression at E13.5 was broader than that of Satb2. In contrast to Satb2, Satb1 mRNA was detected in the whole spinal cord and in two groups of postmitotic cells of the ventral telencephalon (Fig. 3A). Interestingly, Satb1 was strongly expressed in the region of the pons/medulla transition (Fig. 3A). Satb1 signal in the cerebral cortex at E13.5 was mostly confined to the SVZ zone of differentiating cells (Fig. 3B).

At E15.5, within the cortex and SVZ, Satb2-positive cells were detected in the intermediate zone (IZ) and cortical plate (CP) (Fig. 4B and H). Satb1 expression at E15.5 within the telencephalon was detected in the cells of the marginal zone (MZ) and subplate cells of the developing hippocampus (Fig. 4B and H). Satbl was also detected in the piriform cortex and olfactory tubercle (Fig. 4F). Interestingly, medial boundary of Satbl expression in the piriform cortex coincided with the lateral boundary of Satb2 expression in the neocortex

FIG. 5. Satb2 protein distribution and birth dating of Satb2-expressing neurons. (A) Saggital and (B) transverse sections of E11.5 mouse embryos. Satb2 protein is expressed in the cells of lateral nasal process and developing jaws. (E) Satb2 protein distribution in the E13.5 diencephalon. Three groups of Satb2 cells are depicted by arrowheads. (G) Satb2 protein in the E15.5 telencephalon. Satb2 expression in neocortex (C) and spinal cord (D) $24 \mathrm{~h}$ after a BrdU pulse. Doublelabelled cells are absent from both the telencephalon and the spinal cord. Abbreviations: Aa, amygdala; $\mathrm{cp}$, cortical plate; Hth, hypothalamus; iz, intermediate zone; ln, lateral nasal process; lv, lateral ventricle; ms, mandibular swelling; pcx, piriform cortex; sp, spinal cord; Th, thalamus.

FIG. 6. Colocalization of Satb2 protein with Tbr1, reelin and calbindin in the neocortex. IHC with polyclonal anti-Satb2 (A) and anti-Tbr1 (B) antibody at E15.5. $\mathrm{C}$ and $\mathrm{D}$ are high-magnification views of the boxed regions shown in A and B. Double IHC with polyclonal anti-Satb2 and monoclonal anti-reelin (E) and anticalbindin (F) antibodies at P0. 

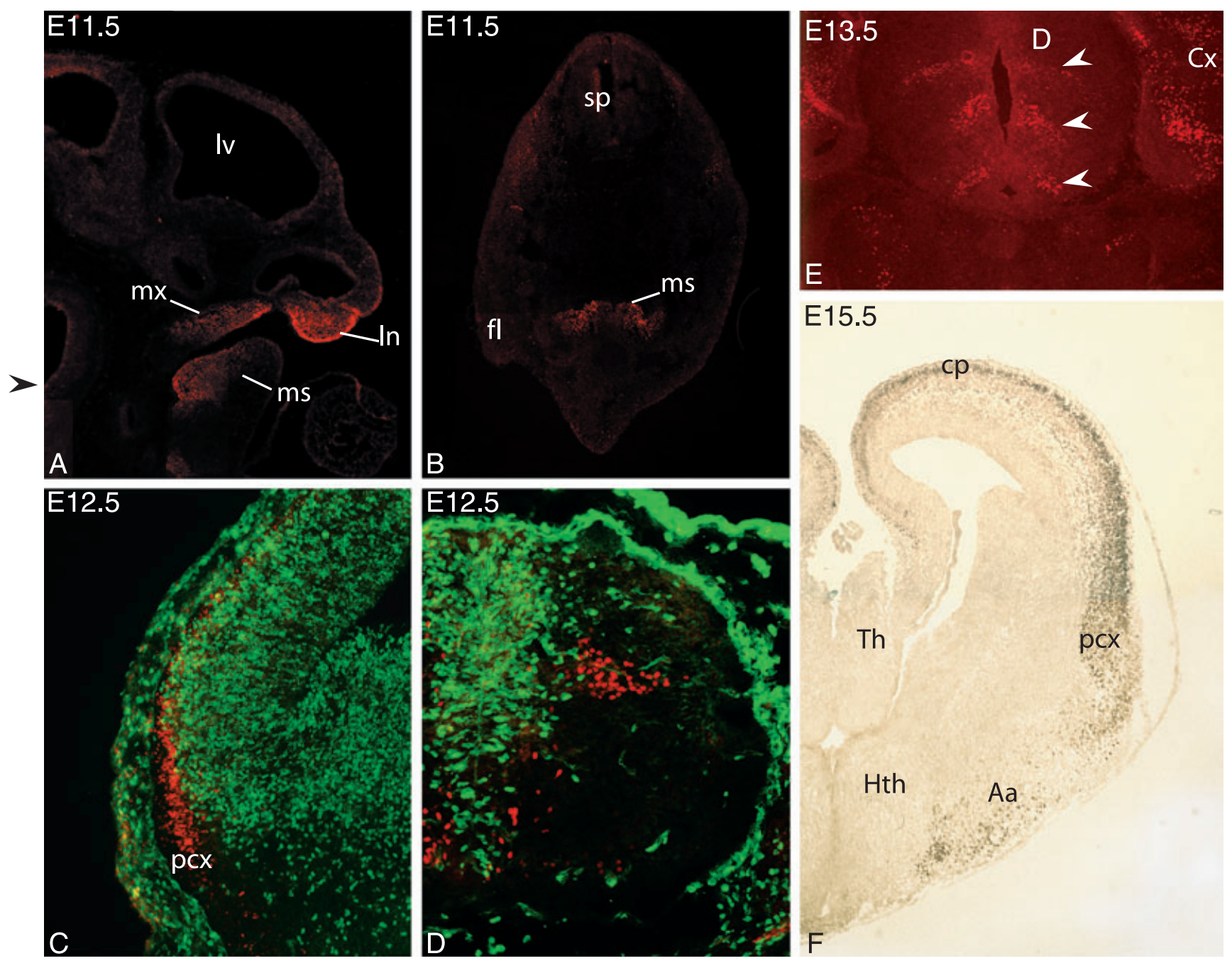

E15.5
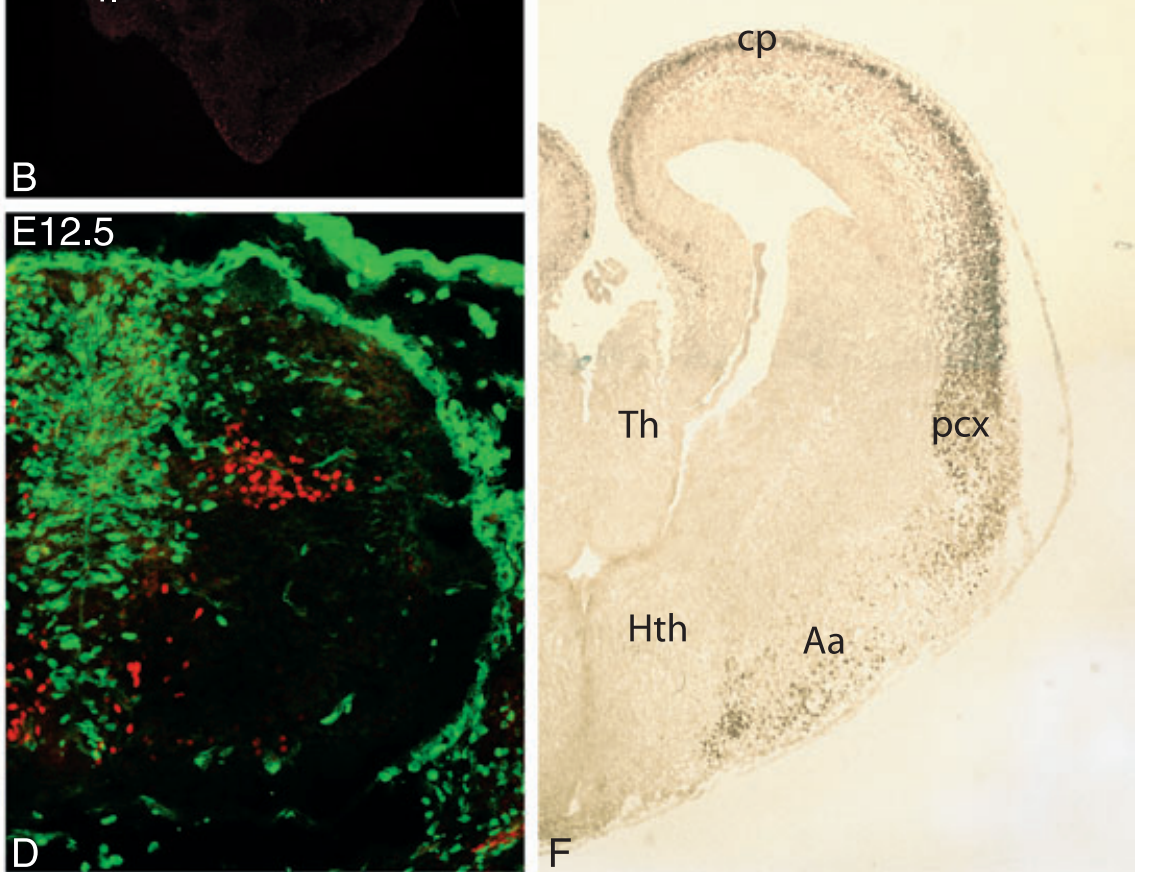

FIG. 5.

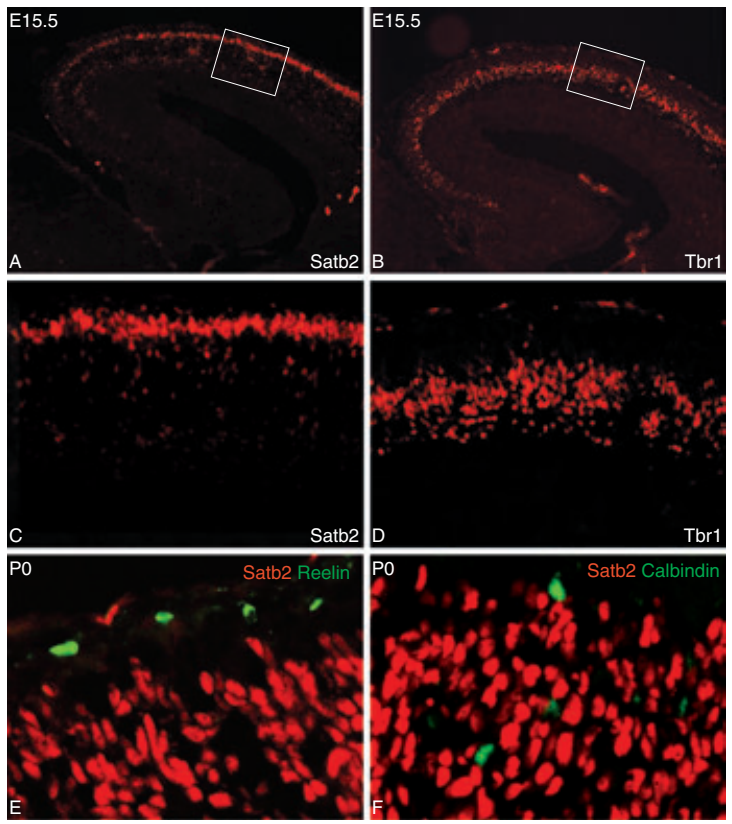

FIG. 6. 
(Fig. 4J-M). This boundary also coincided with the anatomical boundary between the neocortex and piriform cortex (Fig. 4J and L). By contrast, the medial boundary of Satb2 expression coincided with the anatomical boundary between the subiculum and hippocampus (Fig. 4A and B). Expression patterns of Satb2 and Satb1 did not overlap in the telencephalon, indicating that they do not have a redundant function in this tissue.

In order to attribute Satb2 expression to certain subpopulations of the developing telencephalon we compared its expression with the expression of other molecular markers. These markers included reelin as a marker of Cajal-Retzius cells, calbindin as a marker of some GABAergic cells, Dlx 1 as a marker of basal ganglia born interneurons (Anderson et al., 1997), Tbr1 as a marker of neocortical deep layers (Hevner et al., 2001), Pax6 as a marker of the pallial ventricular zone (VZ) and Svet1 as a marker of the SVZ. Expression of Satb2 neither overlapped with the expression of Pax6 nor overlapped with the expression of Dlxl (Fig. 4B, D and E). While Pax6 signal in the pallium was largely confined to the VZ, Satb2 signal was detected in the SVZ, IZ and CP. Expression of Satb2 in the neocortical SVZ partially overlapped with the expression of Svet1. Svet1 expression labels the whole SVZ at E13-E15 (Tarabykin et al., 2001). In contrast, Satb2 mRNA within the SVZ was detected largely in its upper parts (Fig. 4J-I). Comparing their expression at latter stages we observed that while Svet-positive cells stay in the SVZ until E17.5 (Tarabykin et al., 2001), a significant portion of Satb2-positive cells begin migration towards the cortical plate as early as E14.5 (data not shown). At E15.5 many Satb2-positive cells have already reached the CP (Fig. 4B and H). By E18.5 the majority of Satb2-positive cells occupy the upper part of the CP (data not shown).

Double immunohistochemistry (ICH) with anti-Satb2 and antireelin antibodies at E15.5 and postnatal day $(\mathrm{P}) 0$ did not reveal any double positive cells (Fig. 6E). Reelin-positive cells were located in the MZ, which was free from Satb2-positive cells. Most of the calbindin-positive cells did not express Satb2 either (Fig. 6F), although we did detect a few cells weakly labelled by both calbindin and Satb2 (data not shown). We also compared the expression of Satb2 and Tbr1 proteins on adjacent sections at E15.5. Most of the Satb2-positive cells were detected in the upper part of the CP whereas Tbr1-positive cells occupied the deeper part of the CP (Fig. 6A-D). These data indicate that most of the Satb2-positive cells do not coexpress Tbr1, although it is possible that there is a minor portion of Satb2 cells that could also express Tbr1.

ICH staining for Satb2 protein at E15.5 showed that roughly half of the neurons of the CP contained Satb2 protein (Fig. $5 \mathrm{~F}$ ). Satb2 protein, in contrast to mRNA, was not detected in the neocortical SVZ cells (Fig. 5F). These results suggest either a postranscriptional regulation of Satb2 expression or a lower sensitivity of ICH detection as compared with in situ hybridization. Such a difference was not detected for other parts of the CNS expressing Satb2. In the developing diencephalon there were three groups of Satb2-positive cells: ventrally, medially and dorsally (Fig. 5E). In the adult brain Satb2 was expressed in the neocortex and hippocampus although its expression is decreased as compared with that in the embryonic brain (Fig. 4O).

\section{Birth-timing of Satb2-positive neurons in the telencephalon and spinal cord}

In order to determine the birth-time of Satb2-positive neurons we injected pregnant females with BrdU at E11.5. The embryos were taken for IHC analysis $24 \mathrm{~h}$ later. Embryo sections were subjected to double IHC with anti-BrdU and anti-Satb2 antibodies. We did not detect any double positive cells in the neocortex or in the spinal cord (Fig. 5C and D). Satb2-positive cells in the telencephalon were located mostly in the lateral cortical plate directly above the layer of BrdU-positive cells (Fig. 5C). A similar situation was observed in the spinal cord - all the Satb2-positive cells were situated in the MZ outside the domain of BrdU-positive cells in the VZ (Fig. 5D). These results indicate that Satb2-positive cells in the spinal cord and early born Satb2-positive cells in the telencephalon are generated before E11.5.

\section{Satb2 expression in the dorsal spinal cord identifies a subgroup of Lbx1-positive neurons and is abolished in Ibx1 mutants}

The first Satb2-expressing cells in the spinal cord were detected at E12.5 and were observed at all axial levels of the spinal cord. Three domains of Satb2 expression - dorsal, medial and ventral - can be distinguished at this stage within the spinal cord (Fig. 7E). In order to attribute Satb2 to certain neuronal classes we performed double immunostaining of Satb2 and other spinal cord neuronal markers. Staining with anti-Isl1/2 antibody demonstrated that all ventral Satb2 neurons were also coexpressing Isl1/2. However, not all Isl1/2 ventral neurons were Satb2 positive (Fig. 7C and E). Satb2-positive neurons in the dorsal portion of the spinal cord were not positive for either Is11/2, Lmx1b (Fig. 7) or Evx1 (data not shown). They were, however, positive for both $\operatorname{Lim} 1 / 2$ and Lbx1 proteins (Fig. 7). Notably, Satb2-positive cells were expressing Lbx1 at a lower level than most of the other Lbx1 neurons (Fig. 7G-I). Satb2-positive dorsal neurons were located at the lower boundary of dorsal Lbx 1 expression (Fig. 7). Interestingly, a subset of Satb2-positive cells located at this stage in the upper portion of the ventral spinal cord do not coexpress any molecular marker tested.

In order to study whether Satb2 is situated downstream of Lbx1 transcription factor we studied the expression pattern of Satb2 in the spinal cord of $l b x l-/-$ mutant E12.5 embryos. The amount of Satb2positive cells was significantly decreased in the dorsal part of the spinal cord in the mutants. At the same time, ventral and medial Satb2 expression domains appeared to remain unchanged (Fig. 8).

\section{Satb2 in the cerebral cortex is a part of a nuclear complex interacting with nuclear MARs}

In order to investigate the ability of Satb2 protein to bind MARs, we first selected those MARs that can interact with nuclear extracts from the tissue with the highest concentration of Satb2 protein. Developing E18.5 cerebral cortex was selected as a tissue source on the basis of expression experiments. Figure 9 shows an autoradiogram of gel

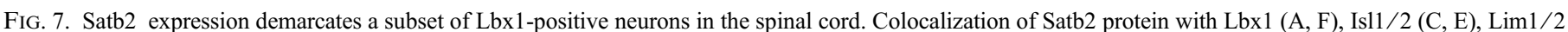

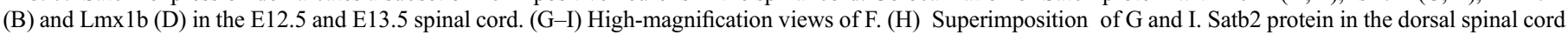

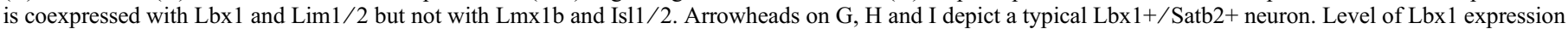

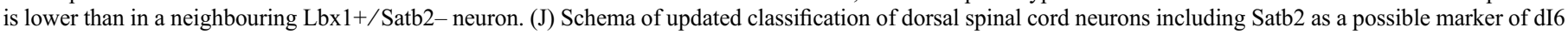
neurons.

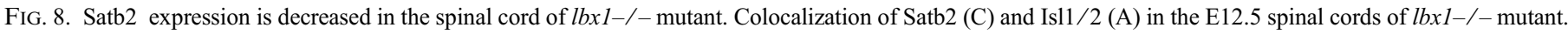

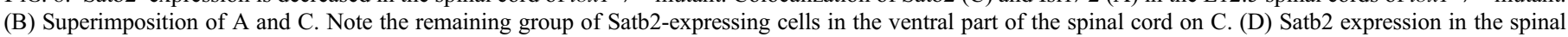

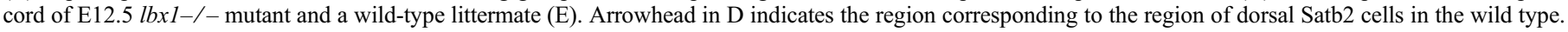



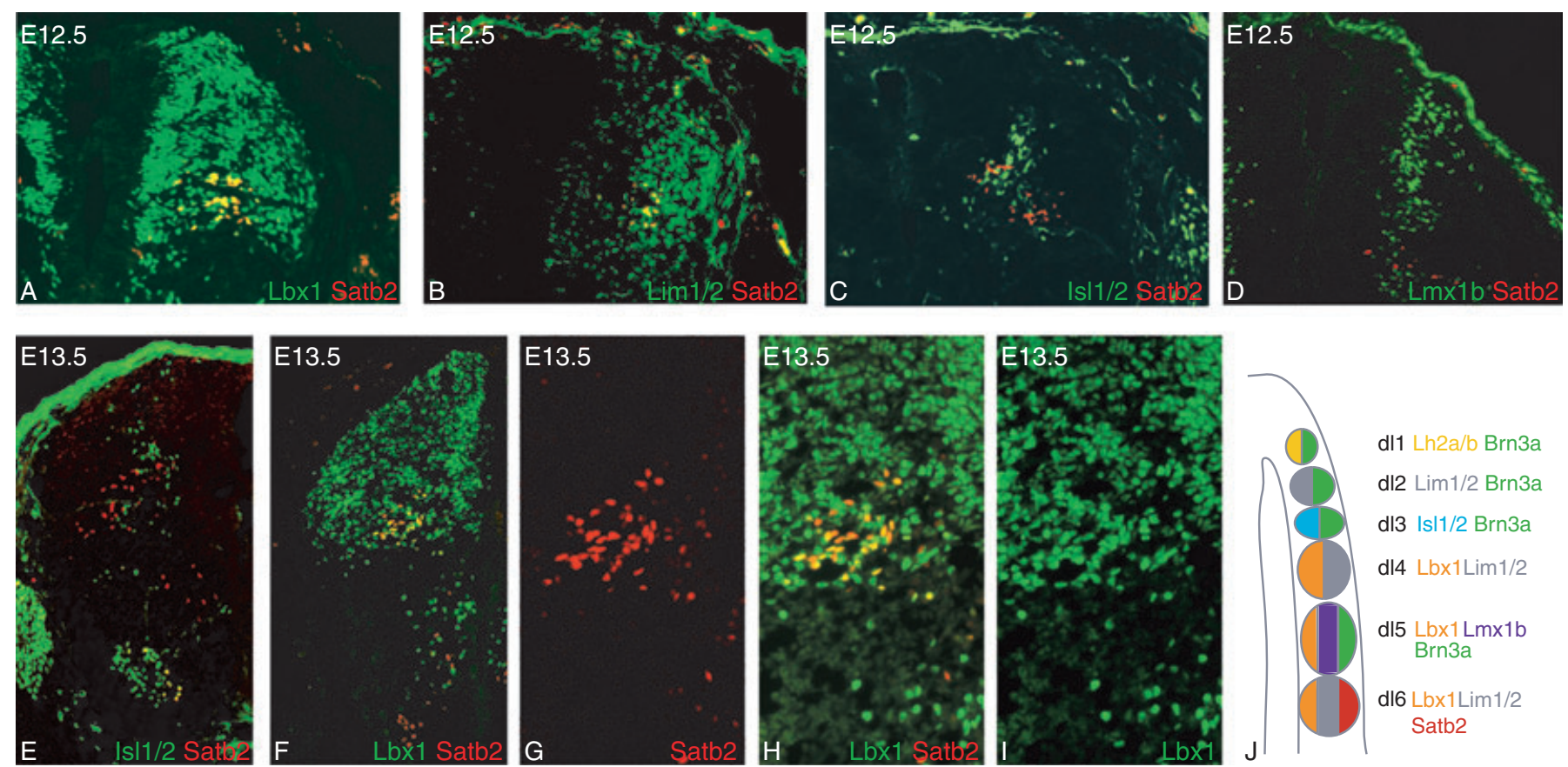

FIG. 7.
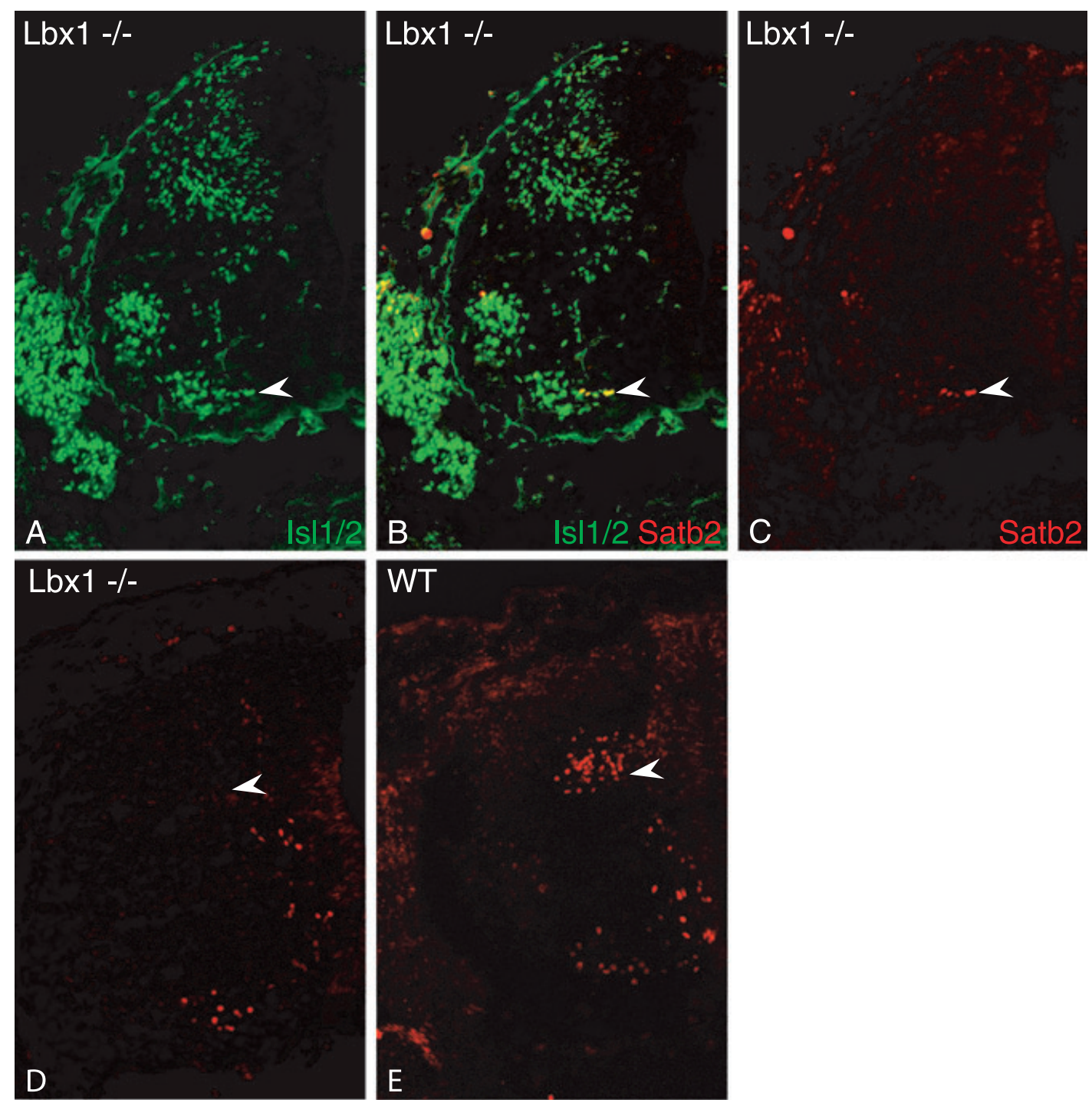

FIG. 8.

(c) 2005 Federation of European Neuroscience Societies, European Journal of Neuroscience, 21, 658-668 


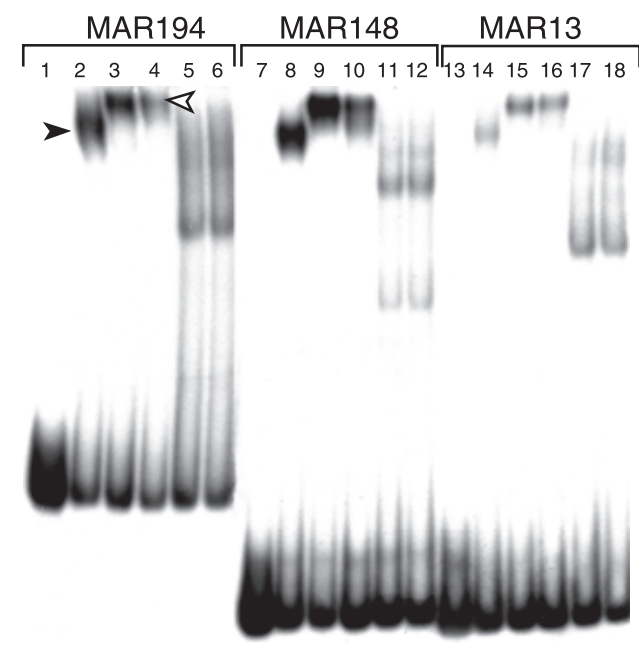

A

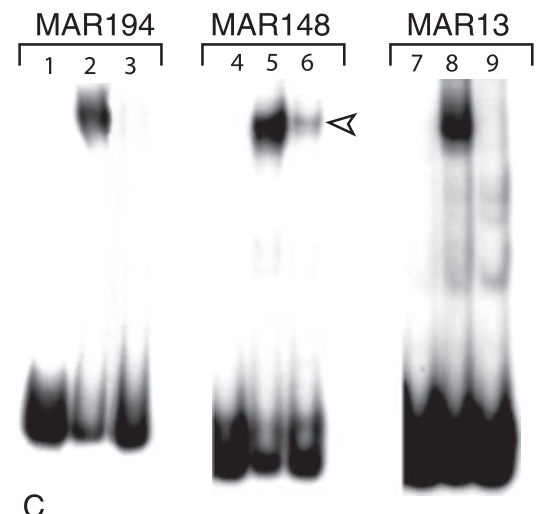

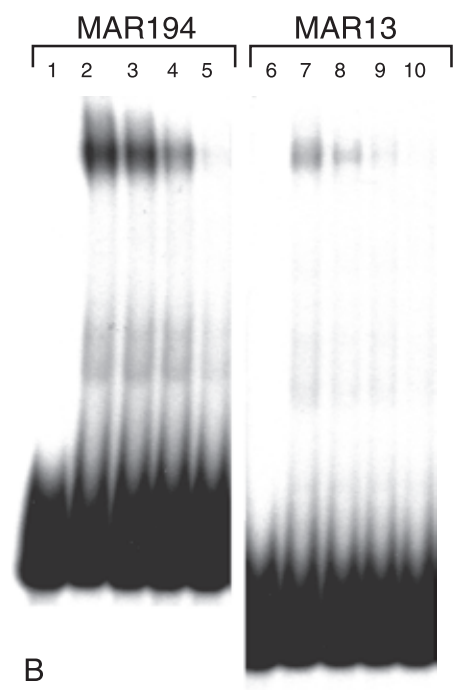

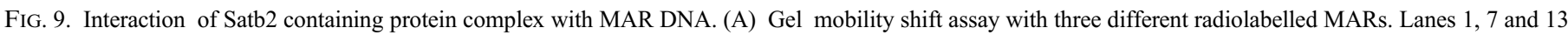

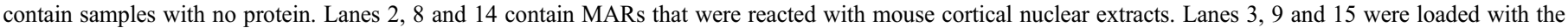

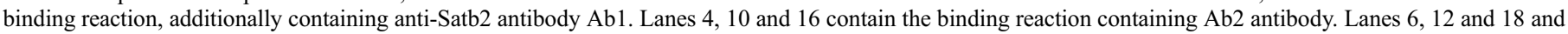

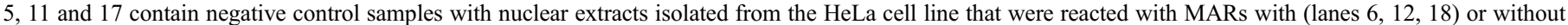

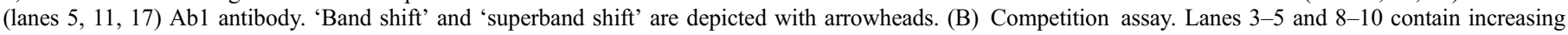

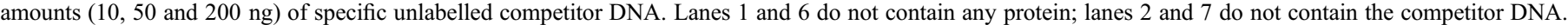

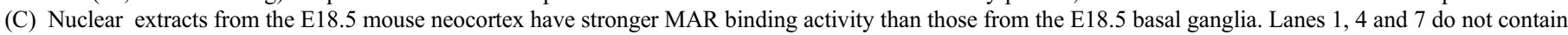

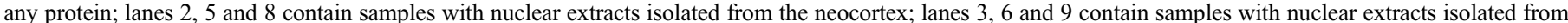

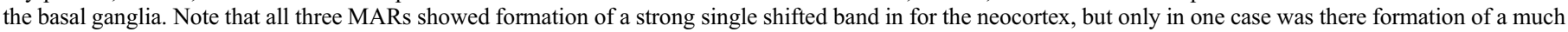
weaker band for the basal ganglia (arrowhead).

mobility shift experiments carried out with hamster and human MARs. The MARs taken for the analysis have been shown to attach to the nuclear scaffold (Nikolaev et al., 1996). In six of ten MAR sequences tested, the addition of nuclear extracts from the cortical tissue resulted in the appearance of a shifted band (Fig. 9A). The shifted bands showed an appearance of a single major binding complex. Interestingly, nuclear extracts isolated from E18.5 basal ganglia tissue for all but MAR148 did not produce strong shifted bands (Fig. 9C). In the case of MAR148 (M2E4) a weaker band of similar size to that of cortical band. Formation of the shifted complex was inhibited by specific unlabelled competitor DNA (Fig. 9B).

We then tested whether Satb2 is a part of the MAR-binding complex in the cerebral cortex. The presence of Satb2 protein in the cortical nuclear extracts was confirmed on a Western blot (Fig. 2) using $\mathrm{Ab} 1$ and $\mathrm{Ab} 2$ antibodies. In order to detect Satb2 within the cortical MAR binding complex, Ab1 antibody was added to the binding reaction. For all three MARs tested this resulted in the supershift of the DNA-protein complex (Fig. 9A). In the negative control experiments the antibody was added to the nuclear extract isolated from HeLa cells, where Satb2 protein was not detected. These experiments, as expected, did not produce any band supershift.

\section{Discussion}

\section{Satb2 expression delineates subsets of differentiating neurons in the developing CNS}

The problem of characterization of different cell types is a common one in many regions of the CNS. The molecular profile of a certain cell type is an important aspect of neuronal phenotype. In this study we investigated Satb2 mRNA and protein distribution with embryonic development. Most of the cells expressing the gene were found within the CNS and developing cartilage tissue. In all regions of the CNS, Satb2 expression peaked in differentiating neurons and decreased in 
mature cells. This suggests that Satb2 is involved in the control of neuronal differentiation. The highest proportion of Satb2-expressing neurons, greater than $50 \%$, was detected in the neocortex between E15.5 and E18.5. In the other parts of the CNS where Satb2 was expressed, such as spinal cord and thalamus, Satb2-positive cells were minor subpopulations comprising not more than $5-10 \%$ of all cells. Interestingly, in the neocortex, Satb2 protein, in contrast to mRNA, was not detected in the SVZ but was detected in the CP. Although this finding could be a result of low sensitivity of IHC, it may also be explained by a Satb2 translation block in the neocortical SVZ cells.

Novel molecular classification of cortical neurons has been suggested recently by Hevner et al. (2003). Cortical neurons were classified on the basis of gene expression profiles. Our data corroborate Satb2 as a valuable marker of cortical superficial layers. Satb2-positive cells do not express reelin, and most do not express Tbr1 and calbindin. Our data also indicate that Satb2 is not expressed in the cells that express another marker of superficial layers and the SVZ, Svet1. However, there may be minor subgroups of cells coexpressing Satb2 and Svet1, or Satb2 and Tbr1. Further experiments will need to be performed to address this issue.

Recently, a new classification of dorsal spinal cord neurons was suggested (Gross et al., 2002; Muller et al., 2002). According to the new classification early dorsal neurons consist of two major classes, Lbx1-negative (class A) neurons and Lbx1-positive (class B) neurons. The latter are subdivided into three classes: D3 (dI4), D4 (dI5) and D5 (dI6). These neurons are distinguished on a basis of the expression of certain combinations of transcription factors that control their differentiation. Two of these groups, however, D3 (dI4) and D5 (dI6), have been undistinguishable by means of molecular markers. Both D3 (dI4) and D5 (dI6) neurons coexpress Lbx1 and Lim1/2. Thus far, they have been distinguished only by their positions along the dorso-ventral axis (Gross et al., 2002; Muller et al., 2002). Our colocalization experiments demonstrated that dorsal Satb2-expressing cells also express Lbx1 and Lim1/2 but do not express Lmx1b or Is11. The fact that dorsal Satb2 neurons are located in the most ventral part of the Lbx1 expression domain suggests that Satb2-expressing cells could be either dI 4 or dI6 neurons. This is also supported by the fact that Satb2-positive neurons in the spinal cord appear before E11.5.

Additionally, we have detected a decrease of Satb2-expressing cells in the dorsal part of the spinal cord of $l b x l-/-$ mutants. It has been shown that inactivation of the $L b x l$ gene in the spinal cord leads to ablation of class B neurons (Gross et al., 2002; Muller et al., 2002). It is not clear whether Satb2 is a direct target of Lbx1, or whether the lack of Satb2-positive cells in the dorsal part of the spinal cord is the result of re-specification of these cells.

\section{Satb2 and Satb1 as potential chromatin remodelling regulators in the developing CNS}

Using the cDNA subtraction approach we isolated and characterized a novel mouse gene Satb2 that encodes a Satb1 homologous transcription factor. We demonstrate that endogenous Satb2 protein is a part of a protein complex that binds to the MARs. It has been demonstrated that Satb1 also binds to MARs and regulates expression of multiple genes in the maturating T-cells. It is suggested that Satbl acts as a global regulator of thymocyte differentiation that provides sites for tissue-specific organization of DNA sequences and regulates regionspecific histone modifications. It has been reported that Satbl is expressed predominantly in thymocytes (Dickinson et al., 1992; Adams et al., 1993) but was also detected in the fetal brain (Adams et al., 1993). We demonstrate here that during development Satbl is expressed in several regions of the developing CNS. Satb2 is also expressed in the developing CNS, but expression patterns of Satb2 and Satb1 do not overlap. In the developing cerebral cortex, for example, Satb1 is expressed in the MZ and piriform cortex whereas Satb2 is not expressed in these cells. Satb2, in turn, is largely expressed in the superficial layers of the neocortex and subiculum. It has been reported that Satb1 knock-out mice have a neurological phenotype such as incomplete eye opening and the clasping reflex (Alvarez et al., 2000). Our results, together with the previous reports, suggest that Satb2 and Satb1 can act as global regulators of cell differentiation in specific cell lineageas within the developing CNS. The molecular basis for Satb1 action was recently demonstrated by Kohwi-Shigematsu and coauthors (Yasui et al., 2002; Cai et al., 2003). They have shown that Satb1 forms a three-dimensional network structure in the thymocyte nuclei and recruits the histone deacetylase into this network. Their results convincingly demonstrate that Satb1 defines a novel class of transcription regulators that function as a protein defining the sites within a chromosome that should be subjected to chromatin remodelling. Satb2 has been recently identified as a gene mutated in human patients with cleft palate. It has also been demonstrated that Satb2 can enhance gene expression and the protein can be modified by sumoylation (FitzPatrick et al., 2003; Dobreva et al., 2004).

The mutually exclusive expression of Satb2 and Satb1 suggest that they control the expression of distinct subsets of genes at the level of chromatin in the developing CNS. Within the CNS there are still cells that do not express either Satb1 or Satb2. Because we did not identify anything other than Satb1 homologues of Satb2 in the mouse genome, it is likely that there should be other proteins similar to Satb1 and Satb2 functioning in other neural cells. It remains to be elucidated whether Satb1 and Satb2 can be substituted for one another or whether each has a unique set of targets. Further experiments involving 'knock-in' experiments need to be performed in order to address this question.

For band-shift analysis we randomly selected ten MARs from the human and hamster genome. Six of these MARs strongly interact with a nuclear protein complex isolated from developing neocortex. We have also demonstrated that endogenous Satb2 protein is a part of the MAR-interacting complex. Note that none of the MARs demonstrated strong interaction with basal ganglia nuclear proteins isolated from the same stage. This surprising finding suggests that the level of chromatin remodelling reactions at E18.5 is higher in the neocortex than in the basal ganglia. It can be explained by the fact that the basal ganglia tissue, although most closely related to the neocortex, is more developmentally advanced at E18.5 and that most of the chromatin remodelling processes could be completed by E18.5. Another possible explanation is that the neocortex, having a higher cellular diversity in general, requires a higher level of chromatin remodelling. The latter hypothesis is also supported by the fact that a histone acetyltransferase gene Querkopf is strongly expressed in the neocortex and is required for normal cerebral cortex development (Thomas et al., 2000).

\section{Acknowledgements}

We are grateful to Manuela Schwark for excellent technical assistance. We thank Drs E. Makarov and O. Makarova, Max-Planck Institute of Biophysical Chemistry, Göttingen, for help with antibody generation, Dr C. Birchmeier, Max-Delbruck-Center for Molecular Medicine, Berlin, for Lbx1 guinea-pig antibody and Lbx1 mutant embryos, Dr T. Jessell, Columbia University, for guinea-pig anti-Lmx1b antibody, Dr M. Sheng, MIT, for Tbr1 antibody, and D. Staroverov, Institute of Bioorganic Chemistry, Moscow, for help with sequencing. O.B. is a recipient of a Humboldt Fellowship. This work was supported by the Max-Planck Society, Volkswagenstiftung grant 78577 (to V.T. and S.L.) and EU grant QLG3-CT-2000-00158 (to P.G.). 


\section{Abbreviations}

$\mathrm{CP}$, cortical plate; ICH, immunohistochemistry; IZ, intermediate zone; MAR, matrix attachment region DNA element; MZ, marginal zone; SVZ, subventricular zone; VZ, ventricular zone.

\section{References}

Adams, M.D., Kerlavage, A.R., Fields, C. \& Venter, J.C. (1993) 3,400 new expressed sequence tags identify diversity of transcripts in human brain. Nat. Genet., 4, 256-267.

Alvarez, J.D., Yasui, D.H., Niida, H., Joh, T., Loh, D.Y. \& Kohwi-Shigematsu, T. (2000) The MAR-binding protein SATB1 orchestrates temporal and spatial expression of multiple genes during T-cell development. Genes Dev., 14, 521-535.

Anderson, S.A., Eisenstat, D.D., Shi, L. \& Rubenstein, J.L. (1997) Interneuron migration from basal forebrain to neocortex: dependence on Dlx genes. Science, 278, 474-476.

Cai, S., Han, H.J. \& Kohwi-Shigematsu, T. (2003) Tissue-specific nuclear architecture and gene expression regulated by SATB1. Nat. Genet., 34, 42-51.

Cockerill, P.N. \& Garrard, W.T. (1986) Chromosomal loop anchorage of the kappa immunoglobulin gene occurs next to the enhancer in a region containing topoisomerase II sites. Cell, 44, 273-282.

Dickinson, L.A., Joh, T., Kohwi, Y. \& Kohwi-Shigematsu, T. (1992) A tissuespecific MAR/SAR DNA-binding protein with unusual binding site recognition. Cell, 70, 631-645.

Dobreva, G., Dambacher, J. \& Grosschedl, R. (2003) SUMO modification of a novel MAR-binding protein, SATB2, modulates immunoglobulin mu gene expression. Genes Dev., 17, 3048-3061.

FitzPatrick, D.R., Carr, I.M., McLaren, L., Leek, J.P., Wightman, P., Williamson, K., Gautier, P., McGill, N., Hayward, C., Firth, H., Markham, A.F., Fantes, J.A. \& Bonthron, D.T. (2003) Identification of SATB2 as the cleft palate gene on 2q32-q33. Hum. Mol. Genet., 12, 2491-2501.

Fry, C.J. \& Peterson, C.L. (2001) Chromatin remodeling enzymes: who's on first?'. Curr. Biol., 11, R185-R197.

Gross, M.K., Dottori, M. \& Goulding, M. (2002) Lbx1 specifies somatosensory association interneurons in the dorsal spinal cord. Neuron, 34, 535-549.

Hevner, R.F., Daza, R.A., Rubenstein, J.L., Stunnenberg, H., Olavarria, J.F. \& Englund, C. (2003) Beyond laminar fate: toward a molecular classification of cortical projection/pyramidal neurons. Dev. Neurosci., 25, 139-151.

Hevner, R.F., Shi, L., Justice, N., Hsueh, Y., Sheng, M., Smiga, S., Bulfone, A., Goffinet, A.M., Campagnoni, A.T. \& Rubenstein, J.L. (2001) Tbr1 regulates differentiation of the preplate and layer 6. Neuron, 29, 353-366.

Izaurralde, E., Mirkovitch, J. \& Laemmli, U.K. (1988) Interaction of DNA with nuclear scaffolds in vitro. J. Mol. Biol., 200, 111-125.

Kikuno, R., Nagase, T., Suyama, M., Waki, M., Hirosawa, M. \& Ohara, O. (2000) HUGE: a database for human large proteins identified in the Kazusa cDNA sequencing project. Nucleic Acids Res., 28, 331-332.

Matz, M., Shagin, D., Bogdanova, E., Britanova, O., Lukyanov, S., Diatchenko, L. \& Chenchik, A. (1999) Amplification of cDNA ends based on template-switching effect and step-out PCR. Nucleic Acids Res., 27, $1558-1560$.

Muller, T., Brohmann, H., Pierani, A., Heppenstall, P.A., Lewin, G.R., Jessell, T.M. \& Birchmeier, C. (2002) The homeodomain factor lbx1 distinguishes two major programs of neuronal differentiation in the dorsal spinal cord. Neuron, 34, 551-562.

Nikolaev, L.G., Tsevegiyn, T., Akopov, S.B., Ashworth, L.K. \& Sverdlov, E.D (1996) Construction of a chromosome specific library of human MARs and mapping of matrix attachment regions on human chromosome 19. Nucleic Acids Res., 24, 1330-1336.

Rebrikov, D.V., Britanova, O.V., Gurskaya, N.G., Lukyanov, K.A., Tarabykin, V.S. \& Lukyanov, S.A. (2000) Mirror orientation selection (MOS): a method for eliminating false positive clones from libraries generated by suppression subtractive hybridization. Nucleic Acids Res., 28, E90.

Schubeler, D., Francastel, C., Cimbora, D.M., Reik, A., Martin, D.I. \& Groudine, M. (2000) Nuclear localization and histone acetylation. a pathway for chromatin opening and transcriptional activation of the human betaglobin locus. Genes Dev., 14, 940-950.

Stoykova, A. \& Gruss, P. (1994) Roles of Pax-genes in developing and adult brain as suggested by expression patterns. J. Neurosci., 14, 1395-1412.

Tarabykin, V., Stoykova, A., Usman, N. \& Gruss, P. (2001) Cortical upper layer neurons derive from the subventricular zone as indicated by Svetl gene expression. Development, 128, 1983-1993.

Thomas, T., Voss, A.K., Chowdhury, K. \& Gruss, P. (2000) Querkopf, a MYST family histone acetyltransferase, is required for normal cerebral cortex development. Development, 127, 2537-2548.

Yasui, D., Miyano, M., Cai, S., Varga-Weisz, P. \& Kohwi-Shigematsu, T. (2002) SATB1 targets chromatin remodelling to regulate genes over long distances. Nature, 419, 641-645. 